\title{
Multiple MEG and Multiple TMS: Two New Electromagnetic Measurement and Induction Techniques
}

Valero $A^{*}$ and Pérez-Sánchez $\mathrm{H}$

Bioinformatics and High Performance Computing, Computer Science Department, Catholic University of Murcia, Murcia, Spain

\begin{abstract}
Neuroscience is a challenging field of science; it is a hybrid sphere of technology, medicine and psychology that will necessarily lay the foundations of the next big technological revolution. Neuroscience will tear down the borders between mind and computer, enabling a two-way interaction that will provide humans with the power of computers, and computers with the creativity and decision-making of human minds. This paper proposes two new techniques for electromagnetic measurement and induction, Multiple MEG and Multiple TMS, which may lead to a new way of interaction between brain and chips. The paper also proposes a theoretical overview in "Computer Neuroscience", focusing on non-invasive synapse measurement and induction, and higher-level signals
\end{abstract}

Keywords: Computer neuroscience; Mind; Computer; Synapse measurement and induction; Brain-machine interfaces.

\section{Introduction}

Neuroscience is a term coined to embrace a hybrid field of technological development and the possibilities that it is opening nowadays can be considered as the greatest step in the evolution of the human species since the origin of life. Not only the possibilities are insondable but also the challenges presented to scientists and physicians are of great difficulty and importance.

The application of the latest advances in computer science and telecommunications of the last few decades opens the door to a brand new source of evolution of human beings in particular and of any living creature in general as genetic evolution will stop being the unique means of evolving. This new source of evolution would come from the application of technology to the development of new psychical abilities of the human mind, and will be a convergence point where technology will go hand in hand with medicine and psychiatry.

There is no doubt the epicenter of these advancements is the human brain. It is the most complex and unknown organ of the human body and it is the basis of the development of the human mind, which is the most challenging goal of Neuroscience. As a first approach, communication and interoperation between human brain and electronic chips must be achieved. The way to succeed in such a connection is to analyse the communication between neurons as the base of human thinking. Neurons intercommunicate by means of synapse and this mechanism must be addressed as the main enabler of the development of the communication between neurons and chips. Also higher-level brain signals must be addressed, since the complexity of human thinking may involve signal aggregation and processing.

As part of the research of synapse, this neural communication mechanism must be studied in the first place as a mere electrical signal. The first step taken by neuroscience is to measure synapse. Once researchers reach a detailed understanding of how neuron intercommunication takes place in the brain for any human activity. The second logical step is that neuroscience succeeds in inducing artificial signals on the brain's neural tissue. The complete success of both synapse measurement and induction will enable a full two-way interaction between brain and chips.

Nowadays there are several techniques already developed (and others being developed at present) for measuring and inducing synapse on neural tissues, but they have important limitations and drawbacks. Two new techniques mMEG and mTMS are proposed in this paper as an improvement of current technologies. It must be said that depending on the concrete scope of each research work one technique may be more suitable than the others, but MMEG and mTMS provide neuroscience with a degree of flexibility and enhancement of existing technologies that involves an added value which will surely will be a great advantage in most aspects of ongoing and future neuroscientific research.

Notwithstanding it must be highlighted that mMEG and mTMS are proposed as two new techniques that will become a part of the existing set of available techniques. In this paper they aren't regarded as technologies that will substitute and compete with any other technique but as an important complement and enhancement of the current ensemble of existing possibilities in this field of research and they will likely result in an advancement for state-of-the-art neuroscience.

In fact, the approach that is proposed in this paper is not only an improvement of existing techniques but also the basis of mMEG and mTMS is applicable to enhance other techniques in the same way it enhances MEG and TMS. It must be remarked that on the one hand mMEG and mTMS are based on applying the directionality of space sensing technology to the brain level a microscale translation of wellknown macroscale technologies. On the other hand, mMEG and mTMS take the most of signals reinforcement and overlay to succeed in bringing the focussing degree needed in neuroscience even to the goal of finally reaching the neuron level.

But mMEG and mTMS enable new research not only to the neuron level; the flexibility and focusable features they provide allow to dynamically change in size and position the area of the brain to

${ }^{*}$ Corresponding author: Valero A, Bioinformatics and High Performance Comput ing, Computer Science Department, Catholic University of Murcia, Murcia, Spain, E-mail: avalero_research@yahoo.es

Received June 24, 2015; Accepted August 04, 2015; Published August 07, 2015

Citation: Valero A, Pérez-Sánchez H (2015) Multiple MEG and Multiple TMS: Two New Electromagnetic Measurement and Induction Techniques. J Comput Sci Syst Biol 8: 243-248. doi:10.4172/jcsb.1000196

Copyright: @ 2015 Valero A, et al. This is an open-access article distributed under the terms of the Creative Commons Attribution License, which permits unrestricted use, distribution, and reproduction in any medium, provided the original author and source are credited. 
be measured or induced. This way, mMEG and mTMS can help to solve the trouble of the variability in neural firing patterns allowing researchers to easily average measures of broader regions of the brain, what helps to minimize this variability issue. MMEG and mTMS may be considered a single technique that provides neuroscience with both measurement and induction to the neuron level and at the same time to a broader level on an adjustable extended area of neural tissue.

This paper doesn't provide any evaluation, benchmarking or proof of concept that supports its proposals because they are based on well-known principles of Antenna's Theory which don't need further practical demonstration. It's just the combination of several principles and the application of macro-level antennas and space technology to the micro-level of the brain, what adds value to this proposal and makes it ground-breaking.

Finally, apart from the two proposed new techniques, this paper also proposes a theoretical overview in "Computer Neuroscience".

\section{Synapse Measurement}

As synapse is the way neurons intercommunicate, the information it transmits is key in understanding the functioning of the human brain. That is the reason why measuring synapse is the first approach to be made in Neuroscience. The capability of synapse modulation is believed to be the base of human cognitive abilities [1].

As regards to electrical synapse, in order to be able to analyze and interpret the electrical signals that are transmitted from neuron to neuron, it is essential to be able to measure the input and output electrical signals of a single neuron. A living and isolated neuron is needed to conduct experiments on how it responds to different electrical signals. Once this technical improvement is achieved, science will be in a position to find out what the role of every single neuron is, and how all neurons interoperate with each other to work a result out. Whether human brain is based in neural computing or not is a question that will be answered by them.

Both recordings/inductions on ensembles of single brain cells (also known as single units) or on the activity of multiple neurons (also known as multi-units) must be in the scope of brain activity research. A series of studies demonstrated that neuronal readout of tactile stimuli could be uncovered using pattern-recognition algorithms, such as artificial neural networks [2,3]. This leads to the importance of the brain activity in terms of both single units and multi-units.

And even in the case that we are able to understand the synapse between two neurons, a big question remains open: if we have an isolated neuron, it is easier to learn how a neuron responds to different artificial electrical signals but how are the electrical signals belonging to natural thoughts and where are they originated?. Measuring synapse, exactly as it happens in the brain, is essential. Focussing on electrical synapse, there are primarily two main classical techniques for measuring it: Electroencephalography and Magnetoencephalography.

\section{Electroencephalography}

Electroencephalography (EEG) measures electrical synapse. The main advantage is the temporal resolution as it permits resolution in the millisecond domain. This property makes EEG ideal for linking with behavior that dynamically changes over short periods of time: changes in neuronal activation are instantaneously reflected in changes in EEG. Another advantage of EEG over other synapse measuring techniques is that, in contrast to magnetic fields variations several electrical changes happening at the same time in different neurons produce an output signal which adds all the individual neuron-to-neuron electrical fields independently of the orientation of the electrical fields. This allows discriminating sub-signals more easily [4].

Two disadvantages of EEG which are important for Neuroscience are on the one hand that the neural tissue, the scalp and the skull are low-pass filters. This means that measurements with EEG only allow studying low-frequency electrical synapse, as high-frequency electrical variations are absorbed before reaching the EEG sensors. In addition to this also DC (Direct Current) changes in brain electrical activity do not account for significant variance in the scalp-recorded EEG [4].

On the other hand, there is a second main disadvantage in EEGs: today's EEGs are only able to register electrical variations coming not from a single neuron but from a region of at least some millimetres. The former disadvantage despite at first sight it may seem less restrictive is in fact much more limiting since there is no way to measure highfrequency synapse with electrodes placed on the scalp and animals' sub-dural EEGs are different to humans' as the abilities and capabilities differ dramatically. Inherently human and animals' brains translate these abilities and capabilities into different electrical activity. High frequency synapse may be key to understand the information passed on from neuron to neuron since frequency modulation may be one of the different possible signal modulation techniques used by the brain to multiplex information on a single output signal.

\section{Magnetoencephalography}

Every electric flow generates a magnetic field around itself which can also be measured with Magnetoencephalography (MEG). This allows finding out the features of the electric current of origin. Neurons' magnetic fields can be measured within a wider frequency spectrum. High frequencies and DC are not attenuated by the neural tissue, the skull or the scalp as it happens with the electrical field.

MEG has also two main disadvantages. The first one is related to electromagnetic noise. Human activity on Earth generates a great deal of noise that interferes with the weak magnetic fields produced by the brain. Hence, the subject and the measuring equipment must be shielded to avoid noisy interferences. The second disadvantage is more difficult to overcome; it is probably a real limitation of MEG. The measurability of the neuromagnetic field produced by the flow of electric current in cerebral neurons is made more likely by the columnar arrangement of the cortical neurons which leads to the summation and re-enforcement of the field of each of the firing neurons [5]. In other words, neurons must be aligned to avoid their magnetic fields to cancel each other, what limits the MEG utility to the study of Cortex.

\section{A new measurement technique: Multiple MEG}

As regards to MEG recording to the neuron level apart from a much higher sensitivity shielding from the magnetic fields of the rest of the neurons will be a key point. To minimize these biological interferences think of a future MEG that measures neurons' magnetic fields as electromagnetic signals from space are measured by an array of radiotelescopes: the measuring equipment would have a very high directivity and gain and as an array just changing the phase of the antenna's power supply it would be possible to orientate the directivity as desired and receive electromagnetic signals from one particular direction only [6]. This would enable recording electromagnetic fields from a very concrete section of the brain which would have the same lobe form than the antenna's directivity.

If sensitivity, directivity and shielding keep improving the accuracy 
Citation: Valero A, Pérez-Sánchez H (2015) Multiple MEG and Multiple TMS: Two New Electromagnetic Measurement and Induction Techniques. J Comput Sci Syst Biol 8: 243-248. doi:10.4172/jcsb.1000196

of MEG will be higher. This will minimize the need of alignment of the neurons to be measured.

In addition to reaching a neuron level accuracy it is also possible to measure MEG by several arrays of antennas at the same time. As stated before an array of antennas would allow researchers to achieve more accurate MEGs apart from being able to orientate easily directivity's lobe from one area of the brain to another [6]. If the signals of several arrays of antennas are added the result would be that there would be several MEGs who would be similar but not identical. If the lobes of the directivity of the arrays are not distant from each other the MEG that is the result of adding the different individual MEG signals would reinforce the electromagnetic signals of that area of the brain where the directivity lobes of each individual MEG array overlay (Figure 1). This sort of MEG (which we can name multiple MEG or mMEG) together with a higher accuracy may lead to overcome the non-linear arrangement of the neurons in the internal areas of the brain and will open the door to measure synapse from inner brain areas apart from the cortex.

\section{Synapse Induction}

After the first approach consisting in measuring synapse and being able to understand how our brain encodes information, Neuroscience will evolve into the generation of artificial neurologic signals and inducing them on neural tissues.

Inducing synapse by means of electrical currents has the aforementioned limitation of the skull, scalp and neural tissue which are low-pass filters. As a result, being able to focus electrical currents on a certain part of the brain is a difficult task. In addition to this due to the three dimensional physical form of the brain, it is difficult to achieve selective synapse induction with external electric currents as Neuroscience should try to be non-invasive. It is also possible to think of installing electrical components within the brain but there are major drawbacks in inserting components in such a delicate organ. And last, EEG-based techniques provide communication channels of limited capacity; their typical transfer rate is currently 5-25 bits/s [7]. Taking into account all these facts and the non-invasive and focusing requirements in particular magnetic induction must be considered as the best way to induce artificially synapse on neurons.

\section{Electromagnetic induction}

Electromagnetic Induction is the most realistic approach to inducing electrical currents on a concrete area of the brain. Its noninvasive nature, in addition to the possibility of concentrating the induction on the desired region of the brain makes it an ideal choice.

By means of coils placed above the scalp an electric current circulating the coil will produce an electromagnetic field that in turn will induce an electric current flowing through the neural tissue. If this induced electric current surpasses the neuron synapse threshold the neuron will be excited and activated. This is what known as Transcranial Magnetic Stimulation or TMS [8].

\section{A new electromagnetic induction technique: Multiple TMS}

The main trouble of TMS is that it induces synapse on a certain area of the brain more concretely all throughout the region on which the induced electric current flows. This may not meet the need of inducing just on a concrete neuron or a small group of neurons. But if thresholds are taken into account it is possible to generate beams that induce several different electric currents so that individually they don't surpass the synapse threshold but where several beams overlay there is induction summation the threshold is surpassed and then the induced electrical current triggers synapse just in the focused neurons.

By making mathematical and engineering calculations coils can be placed on the scalp with a geometrical distribution that allows that this summation happens only on the area where synapse is to be induced. It

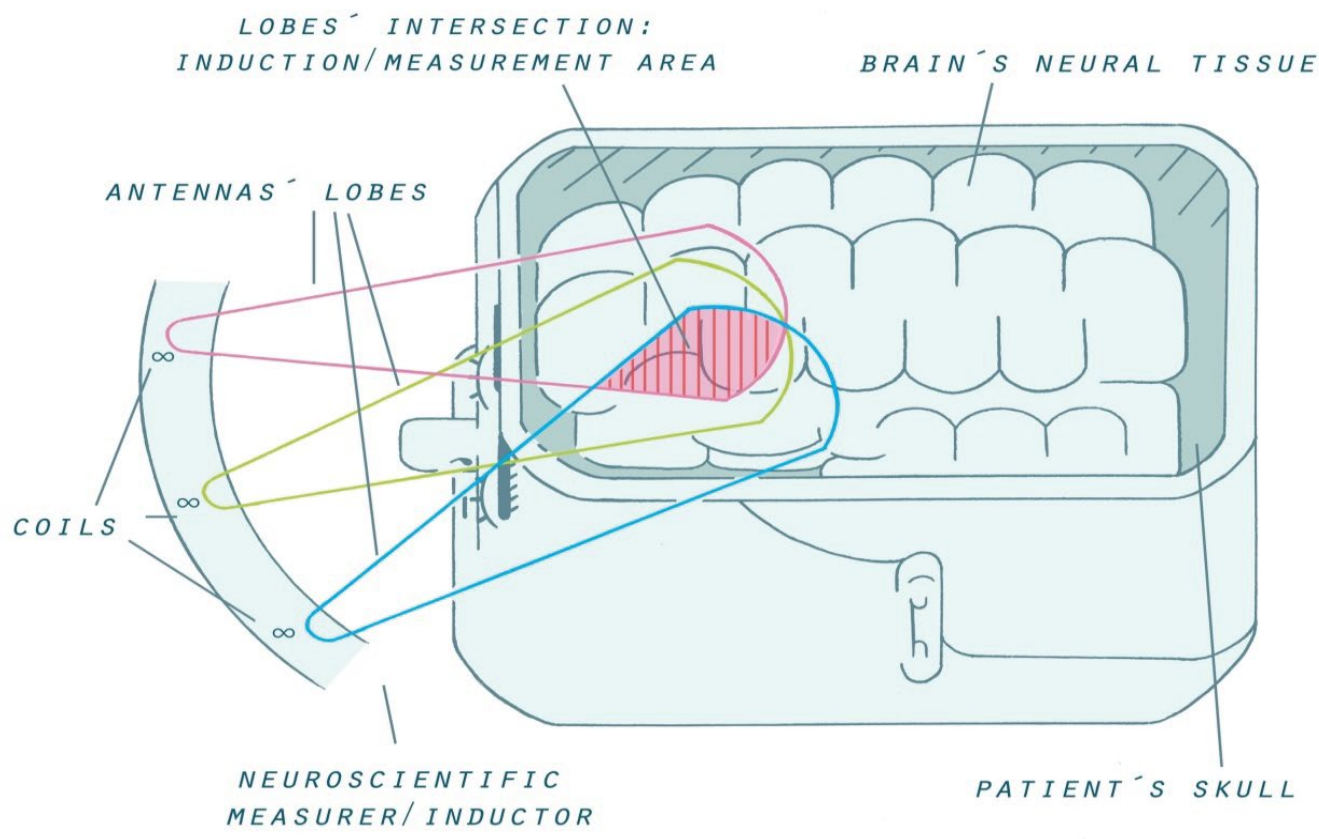

Figure 1: $\mathrm{mMEG} / \mathrm{mTMS}$ neuroscientific measurer/inductor 
must be also considered that it is important to decide the shape of the coil, so that it enables easily summation with the induction of other coils on a predefined area and with big differences between the maximum inducted field value and the average, so that synapse threshold is not surpassed out of the area of summation of the induction of all the coils and hence nearby or middle areas are not excited (Figure 1).

As the accuracy of this sort of TMS (which we can name Multiple TMS or mTMS) improves induction to the neuron level may be achieved, what is an important progress in the induction on noncortical neural tissues where there's no columnar arrangement of the neurons and hence the magnetic fields addressed to different neurons would cancel each other. This mTMS is based on the same idea of the previously proposed mMEG.

Just as it happens with MEG an array of antennas with a higher directivity and gain may be used also for the case of TMS so that just by changing the phase of the antenna's power supply it is possible to orientate the directivity as desired an, together with the proposed mTMS technique induce synapse just in the particular brain area of choice. The electromagnetic principle of reciprocity may even lead to the possibility of using the same arrays of antennas for both measuring and inducing synapse in neural tissues [6].

\section{Related Techniques}

There are multiple techniques for measuring and inducing brain signals. One or another should be chosen depending on the scope of each research and the concrete features of the technique to be selected. Many of these techniques must be seen as complementary approaches and it is difficult to consider that there will be in the future a unique technique which is key to neuroscience.

That being said, we consider that mMEG and mTMS can contribute with important advantages to the set of available techniques in neuroscience. To name just a few advantages which have been already explained in detail in the previous sections mMEG and mTMS may improve dramatically the measurement of electrical activity in much reduced concrete and internal areas of the brain. Another advantage is that they also enable signal induction on neural tissues what undoubtedly will be an important step forward in neuroscience for mind-computer interaction. On top of this the same device can be used either for signal measurement or induction.

Regarding other new techniques to improve neuronal recordings most of them being proposed lately to mention just some examples there are ceramic based multi-electrode arrays [9], nanotechnology devices that reach the brain flowing through the blood stream [10], or techniques such as Low-resolution Electromagnetic Brain Tomography or LORETA [11]. At this point, it should be remarked again that mMEG and mTMS have their own advantages due to their dynamically adjustable area to measure/induct (in terms of both size and position), their non-invasive nature and the added value of being able to use the same device for both measurement and induction.

To sum up either LORETA or any of the other available new techniques leave room for mMEG and mTMS to emerge as additional and complementary techniques in Neuroscience. Finally, the contribution of mMEG and mTMS when compared with MEG and TMS involve that the same approach can be applied to other available techniques bringing similar (or even additional) improvements than those of mMEG and mTMS.

\section{Higher-Level Signals and Future Fields of Research}

As refers to electrical synapse, one of the first issues to consider is what kind of information it transfers and how it is transferred from neuron to neuron. As any other electrical signal, it can't be discarded that synapse can transmit a complex signal which analysed from the point of view of telecommunications may be a modulated and/or multiplexed signal. On the one hand, in the case of electrical synapse it is an electrical signal what is being transmitted and as such not only amplitude but also frequency and phase modulation are possible. Once synapse measurement to the neuron level is achieved reverse engineering techniques can be applied to the signals being analysed so that researchers can find out if in a given recorded synapse signal there's more than just a simple electrical pulse. The point must be to try to trace a carrier signal and what is really important a modulating signal that really may contain useful additional information [12]. On the other hand, assuming the possible existence in the human brain of autonomous regions that compete with each other to give a response to any requirement as this implies the existence of several signals in parallel it can't be discarded that in order to be able to process and take into account the responses from the different autonomous brain areas there is some kind of multiplexing [13]. This would result in the existence of higher-level signals which would be more complex than lower-level synapse signals, and that would contain either the aggregation of simpler signals or the result of higher-level signal processing and signal encoding carried out by the brain.

Apart from measuring and inducing the electrical signals naturally produced by the brain science may discover new capabilities of the human mind unknown to date. These new capabilities would arise when inducing artificial signals that don't exist naturally in the human brain and that would open the door to a brand new field of possible electrical neural responses that would be translated in new sensations, feelings, thoughts or any other kind of brain activity and its corresponding mind-body interaction.

As opposed to current computer architecture components neurons are much more than just a switch with on/off states and an algorithm to handle transitions between these dual states. In fact, an algorithm is based mainly on some input parameters and some output. But an output may be something so faint as a feeling or even any psychosomatic effect. This especially the psychosomatic effects and any mental disease in general lead to the conclusion that in the human body and more concretely in the case of the brain hardware and software are both the same thing and the influence of one on the other is a vague boundary that can't be clearly defined. This involves that when coping with the human mind a new approach must be investigated as current hardware/software conception is not applicable to the brain and its interactions with the rest of the body. When it comes to the mind-body relationship, hardware and software are two sides of the same coin.

The focusable and non-invasive features of mMEG and mTMS will allow researchers to easily measure and induce signals on any region or neuron of the brain. This will let them be able to analyze and reproduce higher-level signals wherever they occur in the brain even in the case that they occur in an inner or especially inaccessible region of the brain. Apart from complete synapse measurement and induction other related disciplines of neuroscience will emerge with more intensity than nowadays such as the study of consciousness how and where it occurs in the brain, what electrical activity it is translated in and how it may be altered with artificially-induced signals. mMEG and mTMS will allow to study deep areas of the brain such as the claustrum where the latest research locates human consciousness [14], 
and where all the information handled by the brain is believed to be merged and processed. The study of the claustrum's electrical activity will likely involve the study of higher-level signals, a question that will be answered when these deep brain areas can be measured and to what mMEG and mTMS will definitely contribute.

\section{Brain-Machine Interfaces and Virtual Reality}

One of the most revolutionary consequences of Neuroscience and that can affect human's way of life more dramatically would be the possibility of building portable brain-machine interfaces (BMIs) in the future.

Before this target can be reached several constraints must be overcome. Among them, the most important for this research is the development of a solution for providing the brain with feedback from a computer [15]. mMEG and mTMS can contribute to this challenging goal together with the progressive miniaturization of the devices needed for them.

The way neurons modulate their responses as a result of movements was already documented decades ago [16,17], but neuron modulations can vary substantially from neuron to neuron and from trial to trial [18-20]. Not only neighbouring neurons might display utterly different firing patterns as a consequence of a particular movement but also single-neuron firing can vary substantially from one trial to the next even in the case that the movements are virtually identical. Notwithstanding, it can be observed that if averages are taken into account there are consistent firing patterns across many trials. In the same way, if the average of a group of neurons is taken into account there is an important reduction in the differences of the signals in comparison to those of single neurons $[18,21]$.

Taking this neuron behaviour variability into account and the way it is reduced when averaging the neurological activity of a broader brain region mMEG and mTMS would provide added value to the set of existing techniques for measuring and inducing neural activity: mMEG and mTMS can be useful to avoid the effects of the variability of signals as they support adjusting the size of the neural area on which they can measure and induce signals. This means that mMEG and mTMS are useful both for neural behaviours that need of a neuron-level resolution and also for neural behaviours whose neuron-level firing patterns variability make necessary averaging signals belonging to a broader area of neural tissue. The variability and dynamical adjustability that mMEG and mTMS support in terms of the position and the size of the area to be measured or induced make these new techniques flexible enough to be applicable to different scenarios and patterns of neural behaviour.

To be assimilated by humans BMI devices will also have to work in the same way and feel the same as the subjects' own limbs. Recent research seems to point to the fact that this assimilation might be succeeded by reproducing an environment in which the brain can experience some kind of plasticity and hence it will assimilate the BMI device as if it were part of its own body. So far, visual feedback has been the most straightforward method to achieve such plasticity. However, the way in which the brain assimilates the BMI device may be improved by using artificial feedback signals produced by sensors installed on the device [15].

BMIs will lay the foundations of a new virtual reality that ultimately will be felt by humans more real than ever. Several strategies have also been proposed to provide feedback to users from BMIs. For instance, virtual-reality systems can provide a realistic feedback that can be efficient for BMI training [22]. There have been recent demonstrations of this kind of proposals where for instance, humans were able to move around in a virtually-generated environment just by imagining they walking [23].

Feedback is key not only for providing the individual with important information in terms of training and decision making (i.e.: use more force to lift an object if it is heavy), but also it is essential for virtual reality systems in a broader sense. The brain is always recalibrating the physiognomy of the body it perceives through the feedback [24]. Hence, thanks to an mTMS-induced feedback, virtual reality systems will be able to mislead the brain and make a virtual body feel as its own physical body.

There are researchers already conducting experiments with third person avatars [25]. Neurological mechanisms may also be the basis for a new field of research in self-presentation [26,27]. Other fields of research could be based on body-image issues where the researchers could perform a controlled manipulation of the self-appearance that the subject perceives visually [24]. mTMS would contribute to these fields of research with both neurological induction and virtual avatars since the technology proposed in this paper would enable at the same time the induction of physical electromagnetic impulses as well as the induction of any computer-generated brain activity related to a simulated avatar.

\section{Conclusions}

In spite of being nowadays almost science-fiction, imagining the future is the best way to know which trail must be followed. In research, finding the right answers is as important as to know which questions must be asked and this paper tries to propose new techniques to wonder about crucial issues and to pose some future fields to be researched in Neuroscience. mMEG and mTMS are very flexible techniques that can provide at the same time focusable measurements and induction of (even deep) brain activity and can interface with neural tissues in terms of both single units and multi-units.

In some decades' time, Neuroscience will turn into state-of-theart technology that will change utterly human way of life not only making it at least sustainable on planet Earth but also opening a brand new world of possibilities ranging from healing mental diseases to expanding human mind's capabilities beyond current natural frontiers.

\section{Conflict of Interest}

The authors declare that they have no conflict of interest, as there isn't any organization that has sponsored the research.

\section{Information Sharing Statement}

This research article includes no data or information resources, neither external nor internal. Hence, the authors don't provide any explicit information sharing statement, as there's no need to disclose any practical sharing details: there are no resources utilized in this work to be accessed by the general public.

\section{References}

1. de Castro LN (2003) Fundamentals of Neurocomputing, Technical Report.

2. Ghazanfar AA, Stambaugh CR, Nicolelis MA (2000) Encoding of tactile stimulus location by somatosensory thalamocortical ensembles. J Neurosci 20 : 3761-3775.

3. Krupa, DJ, Wiest MC, Shuler MG, Laubach M, Nicolelis MA (2004) Layerspecific somatosensory cortical activation during active tactile discrimination Science 304: 1989-1992.

4. Niedermeyer E, Lopes da Silva FH (2005) Electroencephalography: Basic Principles, Clinical Applications, and Related Fields. Congress Cat., USA. 
Citation: Valero A, Pérez-Sánchez H (2015) Multiple MEG and Multiple TMS: Two New Electromagnetic Measurement and Induction Techniques. J Comput Sci Syst Biol 8: 243-248. doi:10.4172/jcsb.1000196

5. Lu ZL, Kaufman L (2003) Magnetic Source Imaging of the Human Brain, Taylor \& Francis, UK.

6. Balanis CA (2012) Antenna Theory: Analysis and Design (3rdedn.) John Wiley \& Sons, USA.

7. Wolpaw JR, Birbaumer N, McFarland DJ, Pfurtscheller G, Vaughan TM (2002) Brain-computer interfaces for communication and control. Clin Neurophysiol 113: 767-791.

8. Kleinjung $T$, Langguth $B$, Khedr E (2011) Transcranial Magnetic Stimulation. In: Møller A, Langguth B, De Ridder D, Kleinjung T (eds.) Textbook of Tinnitus, Springer, New York.

9. Moxon KA, Leiser SC, Gerhardt GA, Barbee KA, Chapin JK (2004) Ceramicbased multisite electrode arrays for chronic single-neuron recording. IEEE Trans Biomed Eng 51: 647-656.

10. Llinas RR, Kerry DW, Masayuki N, Ian H, Patrick AA (2005) Neuro-vascular central nervous recording/stimulating system: using nanotechnology probes. $J$ Nanopart Res. 7: 111-127.

11. Pascual-Marqui RD, Michel CM, Lehmann D (1994) Low resolution electromagnetic tomography: a new method for localizing electrical activity in the brain. Int J psychophysiol 18: 49-65.

12. Carlson AB (2010) Communication Systems (5thedn.) McGraw-Hill, NY, USA.

13. Oppenheim AV (2010) Discrete-Time Signal Processing. Prentice Hall, New Jersey, USA

14. Koubeissi MZ, Bartolomei F, Beltagy A, Picard F (2014) Electrical stimulation of a small brain area reversibly disrupts consciousness. Epilepsy and Behavior 37: 32-35.

15. Lebedev MA, Nicolelis MA (2006) Brain-machine interfaces: past, present and future. Trends Neurosci 29: 536-546.
16. Evarts EV (1966) Pyramidal tract activity associated with a conditioned hand movement in the monkey. J Neurophysiol 29: 1011-1027.

17. Evarts EV (1968) A technique for recording activity of subcortical neurons in moving animals. Electroencephalogr Clin Neurophysiol 24: 83-86

18. Carmena JM, Lebedev MA, Henriquez CS, Nicolelis MA (2005) Stable ensemble performance with singleneuron variability during reaching movements in primates. J Neurosci 25: 10712-10716.

19. Stein RB, Gossen ER, Jones KE (2005) Neuronal variability: noise or part of the signal?. Nat Rev Neurosci 6: 389-397.

20. Cohen D, Nicolelis MA (2004) Reduction of single-neuron firing uncertainty by cortical ensembles during motor skill learning. J Neurosci 24: 3574-3582.

21. Wessberg J, Stambaugh CR, Kralik JD, Beck PD, Laubach M, et al. (2000) Real-time prediction of hand trajectory by ensembles of cortical neurons in primates. Nature 408: 361-365.

22. Bayliss JD, Ballard DH (2000) A virtual reality testbed for brain-computer interface research. IEEE Trans Rehabil Eng 8: 188-190.

23. Pfurtscheller G, Leeb R, Keinrath C, Friedman D, Neuper C (2006) Walking from thought. Brain Res 1071: 145-152.

24. Blom K, Bellido Rivas Al, Alvarez X, Cetinaslan O, Oliveira B, et al (2014) Achieving Participant Acceptance of their Avatars. IEEE Presence 23: 287-299.

25. Kim Y, Sundar SS (2012) Visualizing ideal self vs. actual self through avatars: Impact on preventive health outcomes. Computers in Human Behavior 28 1356-1364

26. Blanke O, Metzinger T (2009) Full-body illusions and minimal phenomenal selfhood. Trends in Cognitive Sciences 13: 7-13.

27. Uddin LQ (2011) Brain connectivity and the self: The case of cerebral disconnection. Consciousness and Cognition 20: 94-98. 